\title{
Refração e seus componentes em anisometropia
}

\author{
Refraction and its components in anisometropia
}

\author{
David Tayah $^{1}$ \\ Marcelo Weslley Lopes Dall' coll ${ }^{2}$ \\ Milton Ruiz Alves ${ }^{3}$
}

\begin{tabular}{|l|}
\hline RESUMO \\
\hline Objetivo: Em anisométropes comparar os valores médios individuais dos \\
componentes oculares de ambos os olhos, correlacionar as diferenças dos \\
componentes com as diferenças de refração; e identificar o menor número \\
de fatores que contenham o mesmo grau de informações expressas no \\
conjunto de variáveis que influenciam a diferença refrativa. Métodos: \\
Realizou-se estudo transversal analítico em população de 77 anisomé- \\
tropes de 2 D ou mais, atendida no ambulatório de Oftalmologia do \\
Hospital Universitário da Faculdade de Medicina Nilton Lins, Manaus. \\
Resultados: Os anisométropes foram submetidos à refração estática \\
objetiva e subjetiva, ceratometria e biometria ultrassônica A-scan. A \\
análise dos dados foi feita por meio dos seguintes modelos estatísticos: \\
análise univariada, multivariada, de regressão múltipla e fatorial. Conclu- \\
sões: Não houve diferenças significativas na comparação dos valores \\
médios individuais dos componentes oculares entre os olhos. Houve \\
correlação negativa média entre a diferença refrativa e a diferença de \\
comprimento axial (r= -0,64) (p<0,01) e correlação negativa fraca entre \\
a diferença refrativa e a diferença de poder do cristalino (r= -0,34) \\
(p<0,01). As variáveis analisadas responderam, no seu conjunto, por 78\% \\
da variação total para a diferença refrativa. Foram identificados três \\
fatores para a diferença refrativa: fator 1 (refração, comprimento axial); \\
fator2 (profundidade da câmara anterior, poder da córnea) e fator 3 (poder \\
do cristalino).
\end{tabular}

Descritores: Anisometropia; Biometria/métodos; Olho/crescimento \& desenvolvimento; Olho/ultrassonografia; Erros de refração

\section{INTRODUÇÃO}

Em relação ao desenvolvimento dos erros refrativos e das anisometropias, muitas pesquisas ${ }^{(1-5)}$ têm procurado responder a diversas questões, como as apresentadas a seguir. Como os componentes oculares influenciam os erros refrativos? Por que alguns indivíduos apresentam anisometropia? Como os componentes oculares influenciam as anisometropias? Tais questões que traduzem preocupações relacionadas com a etiologia, detecção e desenvolvimento da anisometropia, inspiraram a realização desta investigação, conduzida em população de anisométropes de 2,00 D ou mais, com os objetivos de comparar os valores médios individuais dos componentes oculares de ambos os olhos; correlacionar as diferenças dos componentes oculares com as diferenças de refração; verificar a contribuição total e a sequência geral de influência dos componentes oculares na anisometropia e identificar o menor número de fatores que contenham o mesmo grau de informações expressas no conjunto de variáveis que influenciam na composição da anisometropia. 


\section{MÉTODOS}

Trata-se de estudo transversal analítico, conduzido em população de 77 anisométropes de 2,00 D ou mais, calculadas no meridiano vertical, atendida no Ambulatório de Oftalmologia do Hospital Universitário Nilton Lins, Manaus, Amazonas, por demanda espontânea ou referenciada por outros setores do mesmo serviço, no período compreendido entre março e julho de 2007. Os indivíduos incluídos no estudo foram informados de sua natureza e assinaram o termo de consentimento livre e esclarecido.

Foram excluídos anisométropes portadores de qualquer doença ocular; usuários de lentes de contato; suspeitos de ceratocone e/ou submetidos à cirurgia ocular prévia. Todos os participantes foram submetidos à cicloplegia, instilandose uma gota em cada olho, três vezes, com intervalos de 10 minutos, do colírio de cloridrato de ciclopentolato a $1 \%$ (Cicloplégico, Laboratório Allergan Produtos Farmacêuticos Ltda.). Os exames ceratométrico e refratométrico foram realizados 30 minutos depois, empregando-se o refrator automático Topcon KR 8800, programado para a apresentação dos resultados. Foram obtidas três medidas do erro refrativo, e a média foi testada subjetivamente no refrator manual. Os componentes oculares, profundidade da câmara anterior, espessura do cristalino, profundidade da câmara vítrea e comprimento axial, foram medidos com o emprego de um biômetro ultrassônico da marca Tommey, modelo AL 3000, com sonda de frequência de $10 \mathrm{MHz}$, constante de 118,30 e velocidade do som regulada para $1.532 \mathrm{~m} / \mathrm{s}$ para a câmara anterior e cavidade vítrea e $1.640 \mathrm{~m} / \mathrm{s}$ para o cristalino. A técnica de contato foi realizada com o paciente deitado e o examinador posicionado lateralmente. $\mathrm{O}$ valor de cada componente ocular representou a média de três medidas biométricas. Para o cálculo do poder equivalente do cristalino utilizou-se o método de Bennett $^{(6)}$ que requer dados do erro refrativo, poder da córnea, profundidade da câmara anterior, espessura axial do cristalino e profundidade da câmara vítrea.

Dos 77 anisométropes avaliados, 47 (61\%) eram do sexo feminino e 30 (39\%) do sexo masculino. A idade das pessoas incluídas foi de 27,32 \pm 7,96 anos, variando de 9 a 39 anos. Do total, $39(50,6 \%)$ apresentaram anisometropia entre $2,00 \mathrm{D}$ e 3,00 D; $22(28,6 \%)$ entre 2,01 e 3,00 De $16(20,8 \%)$ acima de 3,01 D.

Para a análise dos dados criou-se um banco de dados no Programa Microsoft Access 2000 e foram empregados os programas Statistica for Windows (StarSoft Inc.) release 5.0, 1995; Microsoft Excell 2000 e SPSS for Windows (SPSS Inc.) release 10.0.1, 1999.

Os resultados foram analisados mediante um nível de significância adotado de $5 \%(\alpha=0,05)$, sendo calculados os valores da probabilidade de erro ( $\mathrm{p}$ ) quando $\leq 0,05$ foram considerados estatisticamente significativos $(*)$ e quando $>0,05$ foram tomados como não significativos.

A análise dos dados foi feita calculando-se médias e desvios padrão, medianas, valores mínimos e máximos dos parâmetros biométricos e as comparações entre os olhos foram feitas com o Teste $\mathrm{t}$ não pareado ou com o teste não paramétrico de Mann-Whitney. Para a análise de correlação empregou-se o teste de correlação de Pearson (bicaudal). A análise multivariada foi empregada para examinar a interdependência entre as variáveis e para obter uma equação a fim de predizer a variável refração tendo como base as variáveis componentes oculares. A análise de regressão múltipla foi empregada para explorar relações entre as variáveis. Para a análise fatorial utilizou-se a rotação ortogonal (critério Varimax) para a extração de dimensões não correlacionadas ${ }^{(7)}$.

\section{RESULTADOS}

A tabela 1 mostra os valores da refração do meridiano vertical e dos componentes oculares dos 77 indivíduos anisométropes do estudo.

A tabela 2 mostra os valores dos coeficientes de correlação entre as diferenças dos componentes oculares e as diferenças de refração do meridiano vertical dos indivíduos anisométropes do estudo.

O gráfico 1 apresenta o diagrama de dispersão, a reta interpolatriz e o " $r$ " calculado das diferenças de refração do meridiano vertical com as diferenças de comprimento axial dos indivíduos anisométropes.

O gráfico 2 mostra o diagrama de dispersão, a reta interpolatriz e o "r" calculado das diferenças de refração do meridiano vertical com as diferenças do poder equivalente do cristalino dos indivíduos anisométropes.

A tabela 3 mostra os valores dos coeficientes de correlações múltiplas para os subconjuntos de variáveis das diferenças entre os olhos dos indivíduos anisométropes.

A análise dos dados acima permitiu encontrar a seguinte sequência geral de ordem de importância das variáveis: refração do meridiano vertical, comprimento axial, poder do cristalino, poder da córnea e profundidade da câmara anterior.

As equações de regressão múltipla, calculadas a partir dos coeficientes de correlações parciais e múltiplos das diferenças entre os olhos, foram as seguintes:

(1) RefrMV $=-1,07-1,54 * \mathrm{Cax}-0,31 * \mathrm{PCA}-0,44 * \mathrm{Qt}-0,24 * \mathrm{Pcris}$ (2) $\mathrm{Cax}=0,06-0,29 * \mathrm{RefrMV}+0,08 * \mathrm{PCA}-0,23 * \mathrm{Qt}-0,07 *$ Pcris (3) $\mathrm{PCA}=-0,08-0,01 *$ RefrMV $+0,02 * \mathrm{Cax}+0,13 * \mathrm{Qt}-0,004 *$ Pcris (4) $\mathrm{Qt}=0,02-0,02 * \mathrm{RefrMV}-0,07 * \mathrm{Cax}+0,16 * \mathrm{PCA}-0,004 *$ Pcris (5) Pcris $=1,11-0,65 *$ RefrMV - 0,97*Cax - 0,22*PCA - 0,23*Qt

Como resultado da análise fatorial pelo método de rotação ortogonal (critério Varimax), considerando-se os fatores com "eigenvalue" $>1,0$, foram obtidos três fatores que, no seu conjunto, responderam por $78 \%$ da variação total dos dados para a diferença refrativa entre os olhos. A tabela 4 mostra os três fatores identificados pela análise fatorial.

\section{DISCUSSÃO}

Neste estudo, não houve diferença significativa entre as médias das medidas individuais dos diferentes componentes 


\begin{tabular}{|c|c|c|c|c|c|c|}
\hline \multicolumn{7}{|c|}{ Refração meridiano vertical (D) } \\
\hline Olho de menor ametropia & $-2,60$ & 4,27 & $-1,00$ & 0,00 & $-14,98$ & \\
\hline \multicolumn{7}{|l|}{ Poder corneano médio (D) } \\
\hline Olho de maior ametropia & 43,85 & 1,67 & 43,75 & 39,50 & 49,25 & $0,9251(1)$ \\
\hline Olho de maior ametropia & 3,60 & 0,89 & 3,45 & 1,90 & 5,40 & $0,9525(1)$ \\
\hline Olho de menor ametropia & 3,64 & 0,98 & 3,60 & 1,80 & 5,00 & \\
\hline \multicolumn{7}{|c|}{ Poder equivalente do cristalino (D) } \\
\hline Olho de maior ametropia & 22,75 & 4,90 & 22,79 & 13,31 & 32,92 & $0,2679(1)$ \\
\hline Olho de menor ametropia & 21,73 & 4,42 & 21,82 & 10,88 & 31,67 & \\
\hline
\end{tabular}

\begin{tabular}{|c|c|c|c|c|c|}
\hline & RefrMV & Qt & PCA & Cax & Pcris \\
\hline RefrMV & 1 & $-0,04$ & $-0,12$ & $-0,64\left(^{* *}\right)$ & $-0,34\left({ }^{* *}\right)$ \\
\hline Qt & & 1 & 0,14 & $-0,06$ & 0,03 \\
\hline PCA & & & 1 & 0,10 & $-0,01$ \\
\hline Cax & & & & 1 & 0,00 \\
\hline Pcris & & & & & 1 \\
\hline \multicolumn{6}{|c|}{$\begin{array}{l}\text { RefrMV= refração do meridiano vertical; Qt= poder central da córnea; PCA= } \\
\text { profundidade da câmara anterior; Cax= comprimento axial; Pcris= poder } \\
\text { equivalente do cristalino. } \\
\left({ }^{* *}\right)=\text { correlação de Pearson significativa para nível descritivo de } 0,01 \\
\text { (bicaudal). }\end{array}$} \\
\hline
\end{tabular}

oculares de ambos os olhos dos indivíduos anisométropes (Tabela 1). Em olhos com erros refrativos entre $+6,00$ e $-4,00 \mathrm{D}$, alguns autores ${ }^{(8)}$ não encontraram diferenças nos valores dos componentes oculares medidos em olhos emétropes e amétropes e verificaram distribuição normal de todos os componentes oculares. Nesta investigação, os componentes poder corneano, profundidade da câmara anterior e poder do cristalino mostraram distribuição normal. O comprimento axial não mostrou distribuição normal. Outros autores ${ }^{(9-11)}$ encontraram curva de distribuição normal para o poder corneano, poder do cristalino e câmara anterior. Para o comprimento axial encontraram curva de distribuição leptocúrtica. Baseando-se nesse achado os autores sugeriram a existência de processo adaptativo no qual o comprimento axial seria ajustado pelo poder refrativo ocular. Estudos in vivo têm reportado distribuição normal dos componentes oculares, distribuição leptocúrtica dos erros refrativos e correlação significativa do erro refrativo com o comprimento axial ${ }^{(3,8,12)}$. Tais observações têm levantado a hipótese de que os erros refrativos resultam de mal combinada associação entre os componentes oculares ${ }^{(2-3,5,9,12-20)}$.

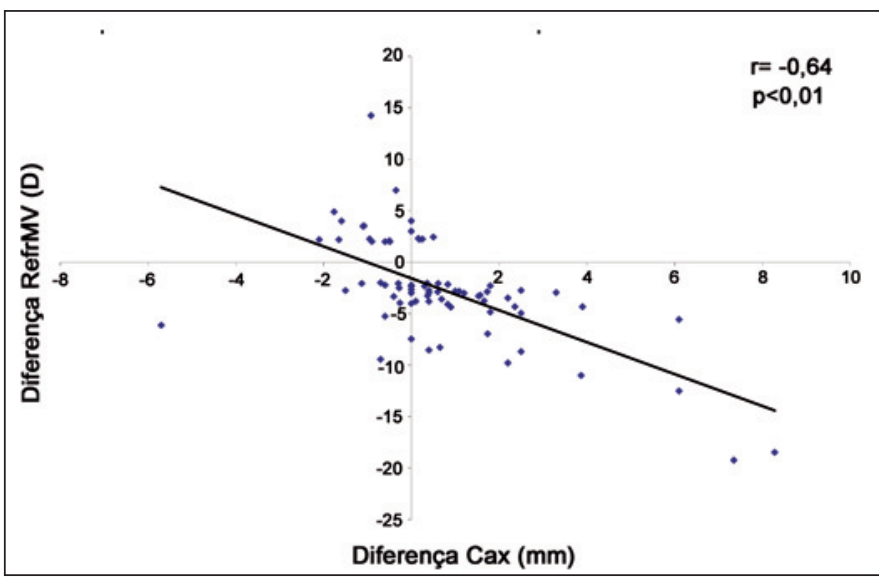

Gráfico 1 - Diagrama de dispersão, reta interpolatriz e "r" calculado das diferenças de refração do meridiano vertical (RefrMV) com as diferença de comprimento axial (Cax) de 77 indivíduos anisométropes. Hospital Universitário da Faculdade de Medicina Nilton Lins, Manaus, Amazonas - 2007.

Neste estudo houve correlação negativa média entre a diferença refrativa e a diferença de comprimento axial $(r=-0,64$; $\mathrm{p}<0,01)$ e correlação negativa fraca entre a diferença refrativa e a diferença de poder do cristalino $(r=-0,34 ; \mathrm{p}<0,01)$ (Tabela 2 e Gráficos 1 e 2). O cálculo do poder equivalente do cristalino foi feito pelo método de Bennett ${ }^{(6)}$. Alguns autores ${ }^{(21)}$ compararam a oftalmofacometria com o método de Bennett ${ }^{(6)}$. As diferenças estimadas entre os dois métodos ficaram entre 0,01 e 0,77 D, com média de diferença de 0,23 D.

Alguns autores avaliaram a variabilidade das medidas de componentes oculares usando a ultrassonografia e encontraram média de variabilidade de $0,062 \pm 0,043 \mathrm{~mm}$ para o comprimento axial; de $0,063 \pm 0,043 \mathrm{~mm}$ para profundidade da câmara anterior e de $0,061 \pm 0,045 \mathrm{~mm}$ para a espessura do cristalino ${ }^{(22)}$. A relação entre comprimento axial e poder 


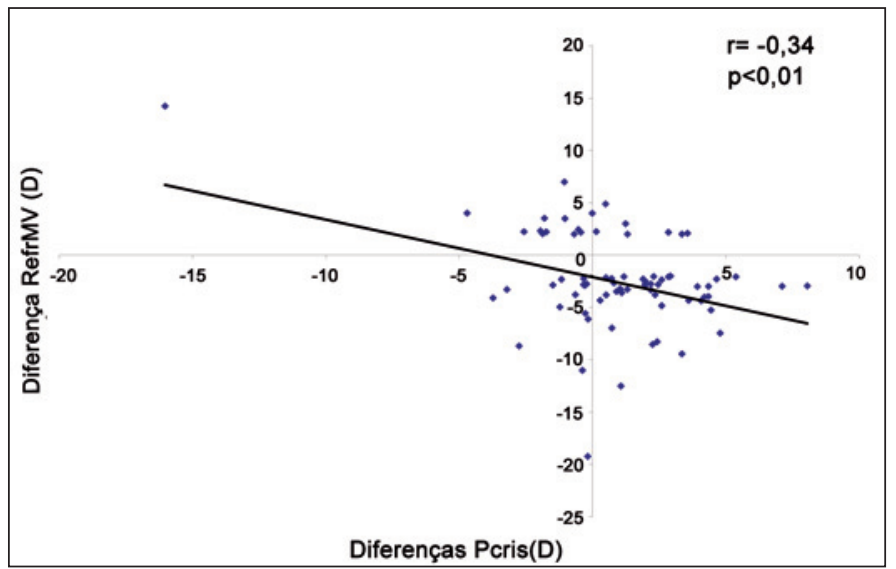

Gráfico 2 - Diagrama de dispersão, reta interpolatriz e o "r" calculado das diferenças de refração do meridiano vertical (RefrMV) com as diferenças do poder equivalente do cristalino (Pcris) de 77 indivíduos anisométropes. Hospital Universitário da Faculdade de Medicina Nilton Lins, Manaus, Amazonas - 2007.

refrativo ocular foi aproximadamente $0,3 \mathrm{~mm}$ de comprimento axial para cada $1,00 \mathrm{D}$, baseando-se no "olho reduzido" de Gullstrand-Emsley ${ }^{(23)}$. A atividade acomodativa aumenta a variabilidade da medida do ultra-som A-scan da profundidade da câmara anterior e do cristalino ${ }^{(24)}$. Diferentes níveis de cicloplegia podem provocar diferenças estimadas de espessura do cristalino ${ }^{(25-26)}$. Pelo fato de a espessura do cristalino poder alterar a profundidade da câmara anterior, o poder equivalente do cristalino calculado a partir da espessura do cristalino e da profundidade da câmara anterior pode também ser afetado, se o cristalino não estiver adequadamente estabilizado pelo agente cicloplégico ${ }^{(24,27)}$. A relação entre espessura e poder dióptrico do cristalino foi de 0,035 a $0,07 \mathrm{~mm}$ de alteração na espessura por dioptria ${ }^{(26,28)}$. A ultrassonografia A-scan foi sensível para variações na espessura do cristalino que excederam 0,86 $\mathrm{D}^{(22)}$. A variabilidade das medidas da profundidade da câmara anterior foi similar à da espessura do cristalino $^{(22)}$. Para minimizar alterações ocasionadas pela acomodação ${ }^{(28)}$, nossos pacientes foram submetidos à cicloplegia com ciclopentolato a $1 \%$, antes do exame ecobiométrico.

A variação total na variável dependente (refração), explicada pela influência das variáveis independentes (poder da córnea, profundidade da câmara anterior, poder do cristalino e comprimento axial), foi verificada a partir dos coeficientes de correlação múltipla. Eles são derivados de coeficientes de correlação parcial e computados para três, quatro ou cinco variáveis, cada variável sendo, por sua vez, considerada dependente de outra. Na tabela 3, temos os seguintes coeficientes de correlação múltipla para o subconjunto de variáveis calculado a partir das diferenças entre os olhos: $1.2345=0,51$; $2.1345=0,46 ; 3.1245=0,04 ; 4.1235=0,04$ e $5.1234=0,15$; sendo $1=$ refração; $2=$ comprimento axial, $3=$ profundidade da câmara anterior, $4=$ poder da córnea e $5=$ poder do cristalino. No subconjunto 1.2345 , a variável 1 ou refração é a variável dependente que sofre a influência das outras variá-

\begin{tabular}{|c|c|c|c|c|c|}
\hline \multirow{2}{*}{\multicolumn{2}{|c|}{$\begin{array}{l}\text { Tabela 3. Coefici } \\
\text { de variáveis calc } \\
\begin{array}{cc}3 \text { variáveis } \\
1.23 \quad 0,41\end{array}\end{array}$}} & \multicolumn{2}{|c|}{4 variáveis } & \multicolumn{2}{|c|}{5 variáveis } \\
\hline & & & & \multirow{6}{*}{1.2345} & \multirow{6}{*}{0,51} \\
\hline 1.24 & 0,42 & 1.234 & 0,42 & & \\
\hline 1.25 & 0,50 & 1.235 & 0,50 & & \\
\hline 1.34 & 0,02 & 1.245 & 0,51 & & \\
\hline 1.35 & 0,11 & 1.345 & 0,11 & & \\
\hline 1.45 & 0,10 & & & & \\
\hline 2.13 & 0,41 & & & \multirow{6}{*}{2.1345} & \multirow{6}{*}{0,46} \\
\hline 2.14 & 0,42 & 2.134 & 0,42 & & \\
\hline 2.15 & 0,45 & 2.135 & 0,45 & & \\
\hline 2.34 & 0,02 & 2.145 & 0,46 & & \\
\hline 2.35 & 0,01 & \multirow[t]{2}{*}{2.345} & \multirow[t]{2}{*}{0,02} & & \\
\hline 2.45 & 0,00 & & & & \\
\hline 3.12 & 0,02 & & & \multirow{6}{*}{3.1245} & \multirow{6}{*}{0,04} \\
\hline 3.14 & 0,03 & 3.124 & 0,04 & & \\
\hline 3.15 & 0,02 & 3.125 & 0,02 & & \\
\hline 3.24 & 0,03 & 3.145 & 0,04 & & \\
\hline 3.25 & 0,01 & \multirow[t]{2}{*}{3.245} & \multirow[t]{2}{*}{0,03} & & \\
\hline 3.45 & 0,02 & & & & \\
\hline 4.12 & 0,01 & & & \multirow{6}{*}{4.1235} & \multirow{6}{*}{0,04} \\
\hline 4.13 & 0,02 & 4.123 & 0,03 & & \\
\hline 4.15 & 0,00 & 4.125 & 0,02 & & \\
\hline 4.23 & 0,03 & 4.135 & 0,02 & & \\
\hline 4.25 & 0,00 & \multirow[t]{2}{*}{4.235} & \multirow[t]{2}{*}{0,03} & & \\
\hline 4.35 & 0,02 & & & & \\
\hline 5.12 & 0,15 & & & \multirow{6}{*}{5.1234} & \multirow{6}{*}{0,15} \\
\hline 5.13 & 0,10 & 5.123 & 0,15 & & \\
\hline 5.14 & 0,09 & 5.124 & 0,15 & & \\
\hline 5.23 & 0,00 & 5.134 & 0,10 & & \\
\hline 5.24 & 0,00 & \multirow[t]{2}{*}{5.234} & \multirow[t]{2}{*}{0,00} & & \\
\hline 5.34 & 0,00 & & & & \\
\hline \multicolumn{6}{|c|}{$\begin{array}{l}\text { variável } 1=\text { refração meridiano vertical; variável } 2=\text { comprimento axial; } \\
\text { variável } 3=\text { profundidade da câmara anterior; variável } 4=\text { poder da córnea e } \\
\text { variável } 5=\text { poder do cristalino }\end{array}$} \\
\hline
\end{tabular}

Tabela 4. Análise fatorial das variáveis considerando-se as diferenças entre os olhos de 77 indivíduos anisométropes

\begin{tabular}{lccc} 
Fator & $\mathbf{1}$ & $\mathbf{2}$ & $\mathbf{3}$ \\
Refração meridiano vertical & $-0,85$ & & \\
Comprimento axial & 0,92 & & \\
Profundidade da câmara anterior & \multicolumn{2}{c}{0,71} & \\
Poder da córnea & 0,79 & \\
Poder do cristalino & & 0,95 \\
fator= refração meridiano vertical, comprimento axial; fator 2= profundidade da \\
câmara anterior, poder da córnea; fator 3= poder do cristalino
\end{tabular}

veis, nesse momento, variáveis independentes. No subconjunto 2.1345, a variável 2 ou comprimento axial é a variável dependente que sofre a influência das outras variáveis independentes. Dessa forma, a importância relativa de qualquer variável independente na variabilidade total da variável dependente pode ser determinada. Na tabela 3, os valores mais baixos para a variável dependente 1 com os das outras três variáveis são mostradas pelo coeficiente $1.345=0,11$. A variável 2 em 1.2345 exerce a maior influência. $\mathrm{O}$ valor mais alto 
mostra $1.245=0,51$ e, então, a variável 3 em 1.345 é a menos importante. Podemos observar que, quando a refração e o comprimento axial são considerados variáveis dependentes, tem-se boa parte da variabilidade dos dados explicada. Tal explicação está em ordem decrescente de grandeza em poder do cristalino, poder da córnea e profundidade da câmara anterior. Esse raciocínio deve ser repetido para outras variáveis a fim de se obter a sequência geral de influências que, neste estudo foi refração, comprimento axial, poder do cristalino, poder da córnea e profundidade da câmara anterior.

A utilização de equações de regressão múltipla, calculadas a partir dos coeficientes de correlação parciais e múltiplos, permite entender como uma variável é influenciada pelas demais variáveis. Por exemplo, a equação;

$$
\text { [RefrMV= -1,07-1,54*Cax-0,31*PCA-0,44*Qt-0,24*Pcris] }
$$

refere-se à diferença de refração do meridiano vertical ocular entre os olhos. Indica que $1 \mathrm{~mm}$ de alteração no comprimento axial entre os olhos modifica a diferença de refração do meridiano vertical de 1,54 D; que $1 \mathrm{~mm}$ de alteração na diferença de profundidade da câmara anterior entre os olhos altera a diferença de refração do meridiano vertical de $0,31 \mathrm{D}$; que $1,00 \mathrm{D}$ de diferença de poder da córnea entre os olhos altera a diferença de refração do meridiano vertical de $0,44 \mathrm{D}$ e, ainda, que $1,00 \mathrm{D}$ de diferença de poder equivalente do cristalino entre os olhos altera a diferença de refração do meridiano vertical de 0,24 D.

A análise fatorial empregada para estudar a estrutura existente entre as cinco variáveis e reagrupá-las identificou três fatores (Tabela 4) que explicam a maior parte da estrutura de correlação do conjunto de variáveis originais, ou seja, responderam por $78 \%$ da variação total dos dados para a diferença entre os olhos.

O fator 1 (refração, comprimento axial) pode estar relacionado com o fato de que olhos de quaisquer tamanhos, hipermétropes no nascimento, têm que se alongar para se tornarem emétropes. Nesse processo de emetropização, o comprimento axial é ajustado pelo poder refrativo total. O grau de ajustamento determina a refração e a forma do bulbo ocular ${ }^{(13)}$. Para uns autores ${ }^{(4)}$, as diferentes correlações observadas no olho portador da menor e da maior ametropia em anisométropes sugerem que o olho mais amétrope falhou em crescer com proporcionalidade.

O fator 2 (profundidade da câmara anterior, poder da córnea) pode estar relacionado com a tendência de olhos mais compridos apresentarem câmaras anteriores mais profundas e córneas mais planas ${ }^{(13)}$. A câmara anterior, embora relativamente profunda ao nascimento, continua se aprofundando à medida que o olho cresce e o cristalino aplana. O seu aprofundamento (aumento da distância entre a córnea e o cristalino) reduz o poder refrativo ocular e contribui para a emetropização ${ }^{(18)}$. A diminuição do poder de córnea, contudo, é insuficiente para compensar o aumento do comprimento axial, possivelmente, devido a sua baixa elasticidade. Por isso, após o nascimento, a córnea cresce relativamente pouco em comparação à esclera, que por expandir-se mais, influencia mais no erro refrativo ${ }^{(29)}$. Para van Alphen ${ }^{(13)}$ não deve haver correlação entre comprimento axial e córnea pelo fato de olhos muito hipermétropes (presumivelmente pequenos) terem córneas mais planas do que se deveria esperar. Esse achado, contudo, não contradiz a regra. Com mais razão, esse fato enfatiza que os erros refrativos resultam de quebra da associação normal entre os elementos refrativos. Assim, podemos esperar córneas mais planas em hipermetropia ${ }^{(18)}$.

O fator 3 (cristalino) pode estar relacionado com o fato de, curiosamente, o poder do cristalino ser independente do tamanho herdado do olho, e consequentemente olhos pequenos podem ter o mesmo poder do cristalino que olhos muito $\operatorname{compridos}^{(13)}$. No entanto, se a emetropia deve ser mantida a despeito do aumento do comprimento axial, então, o cristalino e a córnea devem perder poder. ${ }^{(18)} \mathrm{O}$ cristalino é uma lente flexível dentro de uma cápsula elástica. À medida que aumenta o diâmetro do círculo suspensório, a zônula exerce tensão mecânica na sua circunferência, aplanando-o e diminuindo o seu poder. Ao nascimento, quando a produção de humor aquoso começa e seu volume aumenta, ocorre um rápido aumento de pressão intraocular e então o olho expande e o cristalino aplana ${ }^{(30)}$.

Medidas do poder do cristalino obtidas pela oftalmofacometria em diferentes idades indicam que isto de fato ocorre. A relação entre os diâmetros axial e equatorial do cristalino é de aproximadamente quatro quintos logo após o nascimento, mas aos 4 anos de idade já diminuiu para cerca de um terço ${ }^{(30)}$. Essa associação de aumento de forças intraoculares que aumentam o comprimento axial e aplanam o cristalino não estabelece necessariamente que uma relação causal exista, embora, o fato sugira a existência de mecanismos adaptativos contribuindo para a emetropização ${ }^{(18)}$.

\section{CONCLUSÃO}

Tais achados sugerem, no seu conjunto, falência no mecanismo adaptativo normal em anisométropes, o que poderia traduzir-se não só pela ocorrência de falta de controle ou de "descontrole" do alongamento do comprimento axial (fator 1), mas também pela falência no controle do aplanamento da córnea, do aprofundamento da câmara anterior (fator 2) e do achatamento do cristalino (fator 3 ).

Esses achados abrem novas perspectivas de pesquisas na área em questão, inclusive sobre as diversas condições que interferem no processo de desenvolvimento visual por estimulação inadequada de um ou de ambos os olhos. Tais pesquisas são necessárias para que se possa compreender esses e outros mecanismos envolvidos na gênese da anisometropia.

\section{ABSTRACT}

Purpose: To compare the individual means of ocular components of both eyes in patients with anisometropia; to correlate the differences of the components with refractive differen- 
ces; and to identify the smallest number of factors that contain the same level of information expressed in the set of variables that influence refractive difference. Methods: An analytical transversal study was carried out in 77 patients with anisometropia of two or more dioptres seen at the Ophthalmologic Clinic, University Hospital, Nilton Lins Medical School, Manaus. Results: All participants were submitted to ophthalmologic examination which included objective and subjective cycloplegic refractometry, keratometry and ultrasound biometry. Data analysis comprised the following statistical models: univariate, multivariate, multiple and factorial regression analyses. Conclusions: There were no significant differences in the comparison between the individual means of the ocular components. There was negative correlation between refractive difference and difference of axial length $(r=-0.64 ; p<0.01)$ and weak negative correlation between refractive difference and crystalline lens power difference $(r=-0.34 ; \mathrm{p}<0.01)$. The analyzed variables reached $78 \%$ of the total variation of refractive difference. Three factors were identified for refractive differences: a) factor 1 (refraction, axial length); b) factor 2 (anterior chamber depth, cornea power), and c) factor 3 (crystalline lens power).

Keywords: Anisometropia; Biometry/methods; Eye/growth \& development; Eye/ultrasonography; Refractive errors

\section{REFERÊNCIAS}

1. Gwiazada J, Thorn F, Bauer J, Held R. Emmetropization and the progression of manifest refraction in children followed from infancy to puberty. Clin Vision Sci. 1993;8:337-44.

2. Troilo D. Neonatal eye growth and emmetropisation: a literature revision. Eye. 1992;6(Pt 2):154-60.

3. Sorsby A. Biology of the eye as an optical system. In: Tasmann W, Jaeger EA, editors. Duane's clinical ophthalmology. Philadelphia: Lippincott-Raven; 1995. v.1, p.1-17.

4. Tayah D, Coral-Ghanem V, Alves MR. [The ocular components in anisometropia]. Arq Bras Oftalmol. 2007;70(3):459-64. Portuguese.

5. Brown NF, Koretz JF, Bron AJ. The development and maintenance of emmetropia. Eye. 1999;13(Pt 1):83-92.

6. Bennett AG. A method of determining the equivalent powers of the eye and its crystalline lens without resort to phakometry. Ophthalmic Physiol Opt. 1988;8(1):53-9.

7. Bryan FJ. Multivariate statistical methods. Boston: Chapman \& Hall; 1986. p.72-85.

8. Benjamin B, Davey JB, Sheridan M, Sorsby A, Tanner JM. Emmetropia and its aberrations: a study in the correlation of the optical components of the eye. Spec Rep Ser Med Res Counc (GB). 1957;11(293):1-69.
9. Stenström S. Investigation of the variation and correlation of the optical elements of human eyes. Am J Optom Arch Am Acad Optom. 1948;25(5):218.

10. Araki M. Studies on refractive components of human eye by means of ultrasonic echogram. III. the correlation of refractive components. Acta Soc Ophthalmol Jpn. 1962;66:128-47.

11. Carroll JP. Geometrical optics and the statistical analysis of refractive error. Am J Optom Physiol Opt. 1980;57(6):367-71.

12. Sorsby A, Benjamin B, Sheridan M, Stone J, Leary GA. Refraction and its components during the growth of the eye from the age of three. Memo Med Res Counc. 1961;301(Special):1-67.

13. Van Alphen G. On emetropia and ametropia. Opt Acta (Lond). 1961;142 (Suppl):1-92.

14. Sorsby A, Leary GA, Richards MJ. Correlation ametropia and component ametropia. Visio Res. 1962;2:309-13.

15. Tokoro T, Suzuki K. [Significance of changes in ocular refractive components and development of myopia during seven years]. Nippon Ganka Gakkai Zasshi. 1968;72(8):1472-7. Japanese

16. Larsen JS. The sagittal growth of the eye. 1. Ultrasonic measurement of the depth of the anterior chamber from birth to puberty. Acta Ophthalmol (Copenh).1971;49(3):239-62.

17. Larsen JS. The sagittal growth of the eye. 3. Ultrasonic measurement of the posterior segment (axial length of the vitreous) from birth to puberty. Acta Ophthalmol (Copenh). 1971;49(3):441-53.

18. Mark HH. Emmetropization. Physical aspects of a statistical phenomenon. Ann Ophthalmol. 1972;4(5):393-4.

19. Fledelius HC. Ophthalmic changes from age 10 to 18 years. A longitudinal study of sequels to low birth weight. III. Ultrasound oculometry and keratometry of anterior eye segment. Acta Ophthalmol Copenh). 1982;60(3):393-402.

20. Garner LF, Kinnear RF, McKellar M, Klinger J, Hovander MS, Grosvenor T. Refraction and its components in Melanesian school-children in Vanuatu. Am J Optom Physiol Opt. 1988;65(3):182-9.

21. Dunne MC, Barnes DA, Royston JM. An evaluation of Bennett's method for determining the equivalent powers of the eye and its crystalline without resort to phakometry. Ophthalmic Physiol Opt. 1989;9(1):69-71.

22. Kurtz D, Manny R, Hussein M; COMET Study Group. Variability of the ocular component measurements in children using A-scan ultrasonography. Optom Vis Sci. 2004;81(1):35-43.

23. Michaels DD. Visual optics and refraction: a clinical aproach. St. Louis: Mosby; 1980.

24. Steele CF, Craab DP, Edgar DF. Effects of different ocular fixation conditions on A-scan ultrasound biometry measurements. Ophthalmic Physiol Opt. $1992 ; 12(4): 491-5$.

25. Mutti DO, Zadnik K, Egashira S, Kish L, Twelker JD, Adams AJ. The effect of cycloplegia on measurement of the ocular components. Invest Ophthalmol Vis Sci. 1994;35(2):515-27.

26. Kee CS, Do TC, Lai RY, Wong G, Lam AK. Could a cycloplegic agent be replaced by fogging or a corrective lens in the biometric measurement of the crystalline lens? Ophthalmic Physiol Opt. 1998;18(6):521-6.

27. Storey JK, Rabie EP. Ultrasound: a research tool in the study of accomodation. Ophthalmic Physiol Opt. 1993;3(3):315-20.

28. Garner LF, Yap MK. changes in ocular dimensions and refraction with accommodation. Ophthalmic Physiol Opt. 1997;17(1):12-7.

29. Gernet H. [Compensatory behavior or corneal refraction and bulbar length in buphthalmoss]. Klin Monatsbl Augenheilkd. 1964;144:420-31. German.

30. Barraquer JI, Miguel Varas J Jr. Annotations concerning the relation of forces and pressure in eyes during growth. Ann Ophthalmol. 1971;3(4):425-31. 